\title{
LA FORMATION PROFESSIONNELLE EN FRANCE: ASPECTS HISTORIQUES, CHOIX POLITIQUES, TRADUCTIONS JURIDIQUES ET METHODOLOGIQUES ${ }^{1}$
}

Catherine Guillaumin²

\begin{abstract}
Résumé
Cet article porte sur la formation professionnelle en France et donne les informations nécessaires à la compréhension actuelle du système dans ses dimensions historiques et institutionnelles. Un ensemble de textes organise les parcours professionnels initiaux et continus dans un cadre national valorisant l'alternance et ayant pour finalité l'insertion professionnelle, le maintien en emploi, la réorientation professionnelle, l'autonomie des personnes. Cet outil majeur à la disposition de tous révèle des zones d'ombre et des insuffisances que tentent de pallier des réformes successives.
\end{abstract}

Mots clés: Enseignement professionnel; formation continue; programme de travail et d'études; alternance

\section{FORMAÇÃO PROFISSIONAL NA FRANÇA: ASPECTOS HISTORICOS, ESCOLHAS POLITICAS, TRADUÇÕES JURIDICAS E METODOLOGICAS \\ Resumo}

Este artigo trata da formação profissional na França e fornece as informações necessárias para uma compreensão atual do sistema em suas dimensões históricas e institucionais. Um conjunto de textos organiza as trajetórias de carreira inicial e contínua em uma estrutura nacional, promovendo o estudo do trabalho e com o objetivo de integração profissional, retenção de empregos, reorientação profissional, autonomia pessoal. Essa ferramenta importante, disponível para todos, revela áreas cinzentas e deficiências que as reformas sucessivas estão tentando superar.

Palavras-chave: Educação profissional; educação continuada; programa de trabalho e estudo; alternância

\section{FORMACIÓN PROFESIONAL EN FRANCIA: ASPECTOS HISTÓRICOS, OPCIONES POLÍTICAS, TRADUCCIONES LEGALES Y METODOLÓGICAS \\ Resumen}

Este artículo trata de la formación profesional en Francia y proporciona la información necesaria para una comprensión actual del sistema en sus dimensiones histórica e institucional. Un conjunto de textos organiza las trayectorias de las carreras iniciales y continuas en una estructura nacional, promoviendo el estudio del trabajo y con el objetivo de la integración profesional, la retención laboral, la reorientación profesional, la autonomía personal. Esta importante herramienta, al alcance de todos, revela áreas grises y debilidades que las sucesivas reformas están tratando de superar.

Palabras clave: Educación profesional; Educación contínua; programa de trabajo y estudio; alternancia

\footnotetext{
${ }^{1}$ Recebido em 15/05/2020. Primeira avaliação em 13/06/2020. Segunda avaliação em 30/06/2020. Aprovado em 26/08/2020. Publicado em 25/09/2020. DOI: https://doi.org/10.22409/tn.v18i37.46296

2 Docteur en Sciences de l'Education. Collaboratrice bénévole de l'Equipe de Recherche Education Ethique Santé Agir ensemble et prendre soin EA 7505 de l'Université de Tours. Maître de Conférences (retraitée) de l'Université de Tours.France E-mail: catherine.guillaumin@univ-tours.fr
} 


\section{Introduction}

La loi pour une École de la confiance a été promulguée au Journal Officiel le 28 juillet 2019 : elle abaisse, à l'âge de 3 ans, pour tous les enfants, français et étrangers, l'instruction obligatoire. L'article 15 (qui entrera en vigueur à la rentrée 2020) de la loi concrétise un engagement gouvernemental de stratégie de lutte contre la pauvreté. II prolonge l'instruction obligatoire par une obligation de formation pour tous les jeunes de 16 à 18 ans. Les parents peuvent choisir de scolariser leur enfant dans un établissement scolaire (public ou privé) ou bien assurer eux-mêmes cette instruction.

En France, la formation professionnelle articule historiquement une formation initiale, préparant notamment l'entrée sur le marché du travail, et une formation professionnelle continue destinée aux personnes déjà engagées dans la vie active ou qui s'y engagent. La fin des études initiales correspond conventionnellement à une interruption des études de plus d'un an : la distinction entre études initiales et formation continue dépend du moment auquel la formation intervient dans le parcours de la personne, et non du type de formation suivie.

A l'issue de la classe de $3^{\text {ème }}$ après 9 ans d'études environ en primaire puis au collège, lorsque le jeune a environ 16 ans, trois filières sont proposées, la filière générale, la filière technologique et la filière professionnelle. Ces deux dernières constituent la voie professionnelle et délivrent des diplômes allant du Certificat d'Aptitude Professionnelle (CAP, niveau V, équivalent Cadre Européen des Certifications - EQF European Qualification Framework- CEC1) au titre d'ingénieur (niveau I, CEC 8) en passant par le Brevet de Technicien Supérieur (BTS, CEC 5).

La formation professionnelle est un processus d'acquisition de connaissances et de compétences requises dans des métiers spécifiques ou plus largement sur le marché de l'emploi. Ce processus peut débuter lors de la formation initiale et/ou se poursuivre en formation continue. Aujourd'hui, en France, 4 voies d'accès aux certifications sont instituées : formation initiale sous statut scolaire ou formation initiale sous statut d'apprentissage, formation continue et validation des acquis de l'expérience (Pair, C. 2003) (Breton, 2018).

Ainsi, le champ de la formation professionnelle se compose de deux ensembles relativement autonomes l'un envers l'autre: la formation initiale qui concerne les jeunes sous statut scolaire à temps plein et les apprentis et la formation professionnelle 
continue qui concerne tous les individus ayant quitté ou terminé leur formation initiale ainsi que les adultes sur le marché du travail. L'état actuel est le résultat de transformations incessantes. Cette voie professionnelle, qui se distingue par son objectif d'insertion dans l'emploi, est confrontée aux changements économiques accélérés. Elle est tendue entre plusieurs finalités : adéquation entre formations et emplois et développement de l'autonomie des personnes favorisant l'insertion et la mobilité sociales.

\section{La scolarisation de la voie professionnelle}

Traditionnellement, le métier est appris de manière informelle: l'apprenti acquiert les compétences demandées, par la pratique auprès du maître et des compagnons, en observant et en participant à des tâches de plus en plus complexes. L'industrialisation transforme au cours du XIXème siècle la formation à l'emploi. La division des tâches ne nécessite pas davantage de savoirs que d'être capable d'écrire, lire et compter. L'encadrement est formé par les contremaîtres (anciens ouvriers particulièrement compétents) et les ingénieurs (issus d'une autre classe sociale et ayant reçu une formation technique supérieure) (Pair, 2003, pp. 174-178). De très nombreux auteurs (Prost, 1992, Brucy and Troger 2000, Lelièvre, 2004) s'accordent pour dire que le modèle scolaire caractérise et domine la formation professionnelle en France pendant une longue période. Ils situent le début de cette scolarisation de la formation professionnelle en 1880 lorsqu'après avoir achevé la constitution de l'enseignement élémentaire et sa laïcisation, les républicains promulguent une loi considérée comme l'origine de l'enseignement technique.

La loi Astier en 1919 institue le Certificat d'Aptitude Professionnelle (CAP) et pose les fondements de la formation professionnelle conçue comme vecteur de la promotion sociale (Pigassou, 2004). Cette loi est très importante au sens où elle est la première pierre de la Formation Tout au Long de la Vie (FTLV). Elle permet de certifier la qualification acquise et de construire un début de standardisation des critères de qualification à l'échelle nationale. Les apprentis obtiennent un temps de formation en dehors de l'entreprise, pendant le temps de travail. Brucy \& Troger (2000) montrent que les employeurs des industries mécaniques profitent de cette opportunité pour ouvrir des écoles d'entreprises mais constatent que dans les autres domaines, les 
initiatives sont rares. Les débats se multiplient car les orientations s'affrontent et se confrontent, soulignant tout à la fois, l'insuffisance de l'initiative patronale, la misère, le chômage tout autant que la nécessité de disposer d'une main d'œuvre de plus en plus qualifiée.

Dès la fin de la seconde guerre mondiale, la période dite des Trente glorieuses (1945-1975) bouscule le modèle antérieur sur fond de profondes évolutions technologiques, de demande de consommation, d'échanges internationaux. La libération est le moment où l'organisation des formations professionnelles scolarisée est stabilisée. Les Centres d'Apprentissage deviennent des Lycées Professionnels $(L P)$. Ils sont destinés à la formation des ouvriers et des employés qualifiés. Ils deviennent ainsi les concurrents directs de l'apprentissage et des cours professionnels (Brucy \& Troger, 2000), les collèges techniques prennent en 1959 le nom de Lycées Techniques et visent exclusivement la formation des techniciens. Leur formation n'a pas de visée professionnelle directe. Les instructions de 1946 précisent les finalités de la formation dispensée pour l'ensemble du territoire national. Le terme apprentissage est alors strictement circonscrit et caractérise une formation professionnelle assurée en totalité ou en partie au sein d'une entreprise. Brucy \& Troger (2000, p. 15) montrent qu'entre 1945 et 1985, l'enseignement professionnel a connu un accroissement ininterrompu jusqu'à scolariser à la fin de cette période plus du tiers des lycéens ; l'enseignement technique a progressé mais de manière moins importante ; les effectifs de l'apprentissage sont faibles et ne progressent pas.

L'enseignement et la formation technique ou professionnelle se déroulent dans les établissements scolaires sans contact avec l'entreprise. II y a cependant des exceptions, l'apprentissage tel que défini plus haut, mais aussi les formations médicales, paramédicales, sociales, celles des ingénieurs au cours desquelles les périodes en milieu professionnel sont intégrées dans la formation mais ne font pas l'objet d'une attention pédagogique particulière.

\section{Transformations profondes et émergence de nouveaux paradigmes}

L'élévation du niveau de formation fait dans les années 70-80 l'objet d'une convergence rare dans l'histoire (Pair, C. 2003) : les durées de formation augmentent tandis qu'apparaît une perte de la correspondance entre niveau de formation et niveau d'emploi. 


\section{Les lois de 1971}

Chronologiquement les trois lois adoptées le 16 juillet 1971 en même temps que la loi Delors portant organisation de la formation professionnelle continue dans le cadre de l'éducation permanente constituent un moment essentiel et fondateur des transformations de la formation professionnelle.

La première loi concerne l'apprentissage. Celui-ci se constitue dès lors comme une voie de formation à part entière, avec une formation en Centre de Formation d'Apprentis (CFA) qui doit totaliser au moins 360 heures par an. Cette disposition selon Moreau (2003) fait glisser l'apprentissage, au sens strict des termes, de la formation pratique à l'alternance et construit une institutionnalisation de la formation professionnelle (Combes, 1986).

La deuxième loi concerne l'enseignement technologique et professionnel. Elle est, pour de nombreux auteurs, particulièrement importante. Appay (1992, p. 257-265) soutient qu'un verrou idéologique vient de sauter lors de l'introduction des séquences éducatives en entreprise mises en place en septembre 1979 dans les lycées professionnels. Progressivement localement sont rédigés des contrats liant les lycées professionnels et les entreprises. Le mouvement s'accélère avec en 1986 avec la création des baccalauréats professionnels qui introduisent des périodes de formation en entreprise entre 12 et 24 semaines sur 2 ans, obligatoires et dont l'évaluation est prise en compte pour l'obtention du diplôme. Celui-ci ouvre la voie de l'enseignement supérieur aux élèves issus de l'enseignement professionnel. Enfin la loi d'Orientation sur l'Education de 1989 fixe comme objectif national d'amener en 10 ans, $80 \%$ d'une classe d'âge au baccalauréat. Elle précise que "les périodes de formation en entreprise sont obligatoires dans les enseignements conduisant un diplôme technologique ou professionnel (Guillaumin, 1997, p. 43) ». Dès 1992, le mot alternance sous statut scolaire est clairement énoncé dans les textes officiels organisant toutes les formations préparant au CAP, BEP, Bac Pro, BTS ... dans les lycées professionnels et technologiques.

La troisième loi porte sur la participation des employeurs au financement des premières formations technologiques et professionnelles.

\section{Organismes, diplômes et qualifications professionnelles}


Les organismes gestionnaires des Centres de Formation d'Apprentis sont des organismes privés (associations, entreprises, etc.), des Chambres de Métiers ou de Commerce et d'Industrie et des organismes publics (lycées, ...). Les ressources d'un Centre de Formation d'Apprentis dépendent essentiellement du versement de la taxe d'apprentissage. C'est la ressource principale à laquelle s'ajoutent la participation de l'organisme gestionnaire, les subventions de l'État ou de la région si la convention de création prévoit un financement. Les Centres de Formation d'Apprentis sont soumis au contrôle pédagogique de l'État, au contrôle technique et financier de la région. Les Etablissements Publics Locaux d'Enseignement (EPLE), c'est-à-dire les lycées professionnels et technologiques accueillent à la fois des élèves en formation professionnelle par alternance sous statut scolaire qui sont sous la responsabilité du chef d'établissement et également des apprentis au sein des Unités de Formation d'Apprentis (UFA) situées dans chaque établissement et réunis en un Centre de Formation d'Apprentis Académique ; les Universités ont des Centre de Formation d'Apprentis Universitaire. Les ministères de l'Education Nationale, de l'Enseignement Supérieur et de la Recherche encouragent le développement de la voie professionnelle, notamment en valorisant la voie par apprentissage dans les EPLE et en favorisant des parcours intégrant alternance par voie d'apprentissage et sous statut scolaire ou universitaire, au lycée et à l'Université. L'apprentissage permet de préparer les diplômes professionnels et technologiques de l'Education Nationale et universitaires : certificat d'aptitude professionnelle (CAP), brevet d'études professionnelles (BEP), baccalauréat professionnel, Brevet de Technicien Supérieur (BTS), Diplôme Universitaire de technologie (DUT), licence professionnelle, master. Les apprentis ont un statut de jeune travailleur salarié en entreprise, sous la responsabilité d'un maître d'apprentissage. Ils ont conclu un contrat de travail. Ils peuvent être accueillis dans la fonction publique. Les deux formes de contrat sont le contrat d'apprentissage et le contrat de professionnalisation qui permettent de concilier travail en entreprise et formation théorique, c'est-à-dire l'alternance entre pratique et théorie.

Le contrat d'apprentissage a pour but d'obtenir un diplôme CAP, BAC Pro, BTS, DUT, Licence, Master, ou un titre à finalité professionnelle inscrit au Répertoire National des Certifications Professionnelles (RNCP), regroupant l'ensemble des titres 
professionnels relevant du ministère du Travail. Le contrat d'apprentissage s'adresse aux jeunes entre 16 et 29 ans révolus voire au-delà de 30 ans, si l'apprenti est reconnu comme travailleur handicapé ou s'il a un projet de création ou de reprise d'entreprise nécessitant le diplôme préparé. II doit être reconnu apte à l'exercice du métier lors de la visite médicale d'embauche. Les jeunes âgés d'au moins 15 ans peuvent souscrire un contrat d'apprentissage s'ils ont accompli la scolarité du collège (de la $6^{e}$ jusqu'en fin de $\left.3^{\mathrm{e}}\right)$.

L'apprentissage prépare, aux métiers de l'alimentation, du commerce de détail, du bâtiment et des travaux publics, à des métiers qui relèvent de tous les autres secteurs d'activité : hôtellerie-tourisme, services à la personne, secteur automobile, électronique, etc. Le temps de formation dans un Centre de Formation d'Apprentis (CFA) est d'au moins 400 heures par an, 800 heures pour le Certificat d'Aptitude Professionnelle en deux ans, 1850 heures pour le baccalauréat professionnel en trois ans. Ce temps est plus court que celui des formations dispensées dans les lycées professionnels ou technologiques mais il ne faut pas oublier que le temps passé en entreprise est aussi un temps de formation. Le CFA dispense les enseignements nécessaires pour préparer le diplôme prévu au contrat d'apprentissage. Les programmes de formation et les épreuves d'examen sont identiques pour les élèves et les apprentis préparant les mêmes diplômes. Au CFA, l'apprenti garde son statut de salarié. La période au CFA est donc rémunérée comme temps de travail. II suit les enseignements prévus dans les programmes et les règlements d'examen. La formation de l'apprenti s'effectue également au sein de l'entreprise pour laquelle il travaille. L'apprenti est placé sous la responsabilité d'un maître d'apprentissage choisi en fonction de ses connaissances professionnelles.

Le contrat de professionnalisation a pour but d'acquérir une qualification professionnelle reconnue : un diplôme ou un titre professionnel enregistré dans le Répertoire National des Certifications Professionnelles, un Certificat de Qualification Professionnelle (CQP), une qualification reconnue dans les classifications d'une convention collective nationale. Le contrat de professionnalisation est un contrat de travail en alternance entre un employeur du secteur privé et un salarié répondant à certains critères qui permet d'associer l'acquisition d'un savoir théorique en cours (enseignement général, professionnel ou technologique) et d'un savoir-faire pratique au sein d'une ou plusieurs entreprises. Le contrat doit être écrit et être un Contrat de 
travail à Durée Déterminée (CDD) ou un Contrat de travail à Durée Indéterminée (CDI). Le salarié bénéficie du soutien d'un tuteur. Le contrat s'adresse aux personnes âgées de 16 à 25 ans, aux demandeurs d'emploi de 26 ans et plus, aux personnes bénéficiaires du Revenu de Solidarité Active (RSA), de l'Allocation Spécifique de Solidarité (ASS), l'Allocation Adulte Handicapé (AAH) ou ayant bénéficié d'un Contrat Unique d'Insertion (CUI).

\section{La formation continue et les dispositifs associés}

La formation professionnelle continue est un outil majeur à la disposition de tous les actifs : salariés, indépendants, chefs d'entreprise ou demandeurs d'emploi. Elle permet de se former tout au long de son parcours professionnel, pour développer ses compétences et accéder à l'emploi, se maintenir dans l'emploi ou encore changer d'emploi. Des dispositifs permettent la reconnaissance des acquis pédagogiques ou professionnels autorisant l'accès à une formation ou à certaines dispenses. Toutes ces formations relèvent de la pédagogie de l'alternance. Différentes catégories d'actions concourent au développement des compétences :

Le Conseil en Evolution Professionnelle (CÉP) constitue pour chaque actif une opportunité de faire le point sur sa situation professionnelle et engager, le cas échéant, une démarche d'évolution professionnelle. II a pour ambition de favoriser l'évolution et la sécurisation de son parcours. II vise à accroître ses aptitudes, ses compétences et ses qualifications, en facilitant notamment son accès à la formation. II s'agit d'une offre de services en information, en conseil et en accompagnement personnalisés de projets d'évolution professionnelle, gratuite, confidentielle et accessible à chaque actif, avec un accueil et un conseil individualisé et personnalisé

Le bilan de compétences permet à son bénéficiaire d'analyser ses compétences professionnelles et personnelles, ses aptitudes et motivations, en vue de définir un projet professionnel ou de formation. II est susceptible de précéder une action de formation.

Le Compte Personnel de Formation (CPF) s'est substitué au Droit Individuel à la Formation (DIF) le $1^{\text {er }}$ janvier 2015, avec reprise des droits acquis sur ce dernier. II permet à toute personne active, dès son entrée sur le marché du travail et jusqu'à la date à laquelle elle fait valoir l'ensemble de ses droits à la retraite, 
d'acquérir des droits à la formation mobilisables tout au long de sa vie professionnelle. L'ambition du Compte Personnel de Formation est ainsi de contribuer, à l'initiative de la personne elle-même, au maintien de l'employabilité et à la sécurisation du parcours professionnel. A titre indicatif, pour 2020, les salariés ayant effectué une durée de travail supérieure ou égale à la moitié de la durée légale ou conventionnelle du travail sur l'ensemble de l'année 2019 acquièrent 500 euros par an pour se former (plafonné à 5000 euros) et pour les salariés peu ou pas qualifiés (niveau BEP, CAP), le montant annuel du crédit CPF est majoré à 800 euros (plafonné à 8000 euros).

La formation continue désigne la formation suivie par des personnes ayant terminé leurs études initiales et qui reprennent des études en vue d'obtenir un diplôme ou une qualification supérieure ou dans un champ différent. Elle favorise l'adaptation des travailleurs à leur poste de travail, participe au développement de leurs compétences, en lien ou non avec leur poste de travail, vise à réduire, pour les salariés dont l'emploi est menacé, les risques résultant d'une qualification inadaptée ainsi qu'à favoriser la mobilité professionnelle. L'employeur est dans l'obligation d'organiser la formation de ses salariés. La loi du 5 septembre 2018 impose aux entreprises de contribuer au financement de la formation professionnelle et de l'apprentissage par une contribution financière devenue unique, calculée sur leur masse salariale. Les fonds ainsi mutualisés permettent la prise en charge des coûts de formation des salariés des Très Petites Entreprises (TPE) de moins de 50 salariés et des coûts des formations en alternance (professionnalisation et apprentissage). Cette contribution est versée à des organismes spécialisés qui sont les opérateurs de compétences organisés par branches d'activité. À partir de 2021, la contribution sera versée à l'Union pour le Recouvrement de Sécurité Sociale et des Allocations Familiales (URSSAF).

La Validation des Acquis de l'Expérience (VAE) est une voie alternative, à la voie scolaire, à l'apprentissage ou à la formation continue des adultes, pour l'obtention des diplômes. Le champ de la formation professionnelle, initiale et continue a été profondément transformé par la loi de 2002 qui l'institue. Elle prend en compte les compétences professionnelles acquises à travers des activités salariées, non salariées et bénévoles, en rapport direct avec le contenu du titre ou du diplôme visé. La durée de l'expérience professionnelle considérée est d'au moins un an Les auteurs s'accordent pour reconnaitre que « le premier des défis est très certainement celui de 
la légitimation de la voie de l'expérience, comme modalité d'accès à parité avec la formation formelle (Breton, 2018, p. 62) ». L'auteur (Breton, 2018) situe l'émergence du nouveau paradigme de l'Education et de la Formation tout au long de la vie au moment de la communication par la Commission des communautés européennes du 21 novembre 2001.

«(...) les publications successives des Lignes directrices européennes pour la validation des acquis non formels et informels par le Centre européen pour le développement de la formation professionnelle (CEDEFOP) ont ensuite contribué à traduire en dispositifs une politique fondée sur une logique de parité entre les formes d'apprentissage dits formel, informel et non formel. Au même moment est crée la Commission Nationale de Certification Professionnelle (CNCP) dont l'une des missions est de gérer le Registre National des Certifications Professionnelles (RNCP) (BRETON, 2018, p. 64)»

Les certifications professionnelles désignent les diplômes et titres à finalité professionnelle, certificats de qualification professionnelle (CQP), blocs de compétences, certificats ou habilitations, délivrés par une autorité compétente après vérification des compétences ou connaissances d'une personne, enregistrés au répertoire national des certifications professionnelles ou au répertoire spécifique. Elles ne doivent pas être confondues avec les notions de formations et de qualifications. Elles visent à sécuriser les parcours professionnels, en permettant une reconnaissance des compétences de la personne. Breton (2018, p. 63) reprenant Maillard (2016, p. 9) relève le paradoxe que constitue cette alternative où dans « un espace d'activités sociales foisonnant » à savoir le monde de la certification française, cette nouvelle voie d'accès crée des dispositifs visant à l'ordonner et le rendre lisible. La très grande originalité de cette voie nouvelle (Breton, 2018, p. 62) « est d'instituer dans la loi la possibilité de faire valider des savoirs non acquis en contexte formel (tels qu'écoles ou organismes de formation)». II a donc fallu produire un énorme travail de définition « de ce que sont des acquis expérientiels, de leurs modes de manifestation dans un parcours et une pratique professionnelle, et des critères permettant de les évaluer (Breton, 2018, p. 64)». Au-delà de ce travail, la question pédagogique de l'alternance entre pratique et théorie reste centrale. 


\section{Et aujourd'hui où en sommes nous ?}

Dresser un bilan du travail réglementaire accompli est particulièrement complexe. II permet cependant de mettre en évidence les avancées autorisant des pédagogies novatrices et inventives concernant la formation professionnelle initiale et continue mais laisse apparaître des zones d'ombre, des insuffisances qui soulèvent des questions centrales portant sur le droit à la formation pour tous.

\section{Un cadre législatif favorable et cohérent}

Comme nous l'avons décrit précédemment, la formation professionnelle initiale et continue est instituée. Elle est reconnue comme un outil majeur à la disposition de tous jeunes et actifs: salariés, indépendants, chefs d'entreprise ou demandeurs d'emploi. Elle permet de se former tout au long de son parcours initial et professionnel, pour développer ses compétences et accéder à l'emploi, se maintenir dans l'emploi ou encore changer d'emploi. Les dispositifs sont pluriels et la loi sur la Validation des Acquis de l'Expérience a enrichi un ensemble de mesures favorisant le développement des compétences des individus. L'alternance et l'apprentissage sont reconnus et valorisés dans les différents parcours de formation.

\section{Zones d'ombre et insuffisances}

Cependant, les différentes statistiques montrent une insertion difficile des jeunes, un accès à la formation continue différencié selon les catégories socioprofessionnelles. Bernard, Minni, Testas (2018) montrent que «les poursuites d'études sont plus fréquentes mais que l'insertion professionnelle est toujours difficile pour les moins diplômés ». Les auteurs mettent en évidence qu'en 2015-2016, 46 \% des jeunes âgés de 14 à 29 ans sont scolarisés (élèves, étudiants ou apprentis). La part de bacheliers dans une génération a progressé et les nombres d'inscrits et de diplômes délivrés dans l'enseignement supérieur se sont aussi tendanciellement accrus. Depuis 2008, les effectifs d'apprentis ont diminué dans l'enseignement secondaire, mais ont continué d'augmenter dans le supérieur; l'apprentissage concerne aujourd'hui près de $5 \%$ de l'ensemble des jeunes de 16 à 25 ans. 
En 2016, $9 \%$ des jeunes de 18 à 24 ans sont considérés comme sortants précoces du système scolaire: ils possèdent au plus le brevet des collèges et ne suivent pas de formation. L'insertion des jeunes sur le marché du travail s'étale entre 1 à 4 ans avec un fort taux de chômage (19,8 \% des actifs en 2016). Les jeunes en emploi récemment sortis des études sont plus souvent en sous-emploi ou en emploi à durée limitée et, bien que plus diplômés, perçoivent des salaires moins élevés.

\section{Une situation en fait paradoxale}

La formation professionnelle continue est un moyen d'améliorer sa situation au regard de l'emploi. De plus en plus de diplômes sont délivrés dans le cadre de la formation continue. En 2016, deux personnes de 18 à 64 ans sorties de formation initiale sur cinq ont suivi dans l'année au moins une formation à but professionnel. Cependant, l'accès à la formation, professionnelle continue ou à la formation non formelle à but professionnel, demeure différencié. Ainsi, les cadres, les plus diplômés, les personnes d'âges intermédiaires et les salariés des grandes entreprises se forment davantage que les autres (Gaini, 2018).

Des lois récentes comme celle du 5 septembre 2018 Pour la liberté de choisir son avenir professionne/ modifient en profondeur la gouvernance et le financement $\mathrm{du}$ système de formation professionnelle français avec la création de France compétences associée à une réforme du financement de la formation professionnelle et de l'apprentissage qui bouscule en profondeur le système antérieur. Ce mouvement se caractérise par la libéralisation du marché à l'ensemble des organismes de formation qui souhaitent dispenser des actions de formation par apprentissage et pour lesquelles un niveau de financement pour chaque contrat sera assuré. L'État reste le garant de la bonne utilisation des fonds publics et des fonds mutualisés dédiés à la formation professionnelle. 


\section{Conclusion}

Ces réformes incessantes de la formation professionnelle sont à l'origine de nombreuses réflexions. L'appel à communication du Réseau des Universités préparant aux Métiers de la Formation (RUMEF colloque du 18-20 mars 2020) propose une synthèse actualisée des problématiques. Ainsi les coordonnateurs scientifiques de ce colloque rappellent que

«(..) depuis 2000 , ce sont ainsi pas moins de quatre accords nationaux interprofessionnels entre 2003 et 2017 et cinq lois entre 2002 et 2018 qui ont vu le jour, proposant, peu ou prou, de rompre avec le passé, chacun de ces accords et lois étant opportunément précédé ou accompagné de rapports invitant à en finir avec ce « système à la dérive » et ses « réformes inabouties ». Ce mouvement de « réforme » s'est accompagné de la création régulière de dispositifs et de mesures tant pour les salariés que pour les demandeurs d'emploi et les moins de 26 ans. Accompagnées d'une sémantique de la liberté du choix, de l'autonomie, les mesures inscrites dans la dernière loi en date ne portent pas uniquement sur la formation continue, mais également l'apprentissage et l'assurance-chômage et redistribuent les activités entre les acteurs, dans une logique de compétences et moins de formation. (...) (RUMEF, 2020)».

Ainsi, au fil des années, des avancées considérables ont été construites permettant entre autres la reconnaissance des acquis formels et informels. Néanmoins de larges zones d'ombre subsistent telles celles propres à la formation, à l'insertion, à la reconversion des plus fragiles, à l'accompagnement au développement de l'autonomie des individus, largement sollicitée dans toutes les formes d'emploi. La réflexion associée à la pédagogie de l'alternance, à la formation des formateurs et pédagogues, constitue à ce titre une opportunité, non pas pour résoudre mais tout au moins pour inventer des réponses pédagogiques plurielles afin de mieux répondre aux besoins des laissés-pour-compte de la formation.

\section{Bibliographie}

APPAY, B. L'établissement de formation, bureaucratie et production. L'alternance : histoire et enjeux. Ministère Du Travail, de l'Emploi et de La Formation Professionnelle, 1982, p. 257-265.

BRETON, H. (2018). La Reconnaissance et la Validation des acquis de l'expérience en France: le paradigme de l'expérientiel à l'épreuve des certifications. In: Formazione Continua e Educazione degli Adulti: le dimensioni formale, non 
formale e informale nel confronto fra Italia e Francia. VOL 13, N. ALL 30, 2018, p. 62-72.

BRUCY, G., and TROGER, V. Un siècle de formation professionnelle en France: la parenthèse scolaire? Revue française de pédagogie 131(1), 2000, p. 9-21.

COMBES, M.C. La Loi de 1971 Sur l'apprentissage : Une Institutionnalisation de La Formation Professionnelle.(15). Formation-Emploi, 1986, p. 18-32.

GAINI, M. Accès à la formation: les concepts changent, les constats demeurent https://www.insee.fr/fr/statistiques/3526074?sommaire=3526086, accès 10-04-2018.

GUILLAUMIN, C. Une alternance réussie en lycée professionnel. Alternances, Développements. Paris: L'Harmattan, 1997.

La formation en alternance en France. In Formazione Continua e Educazione degli Adulti: le dimensioni formale, non formale e informale nel confronto fra Italia e Francia. VOL 13, N. ALL 30, 2017, p. 75-87.

JUNEL B., MINNI, C., TESTAS, A. Des poursuites d'études plus fréquentes et une insertion professionnelle toujours difficile pour les moins diplômés https://www.insee.fr/fr/statistiques/3526066?sommaire=3526086

LELIEVRE, C. Les politiques scolaires mises en examen Douze questions en débat. ESF. Pédagogies

Essais.

Paris,

2004.

MOREAU, G. Le monde apprenti. Paris: La Dispute, 2003.

PAIR, C. Diplôme, qualification et insertion professionnelle: La relation formation/emploi. In Le Système Éducatif En France (Dir. Toulemonde, B.) p. 174178. Les Notices de La Documentation Française. Paris, 2003.

PIGASSOU, J. La formation tout au long de la vie. In: TOULEMONDE, B. Le système éducatif en France. La Documentation Française. Les Notices: Paris, 2004, p. $109-120$.

PROST, A. 1992. Education, société et politiques. Une histoire de l'enseignement en France, de 1945 à nos jours. Seuil: Paris, 1992.

\section{Sites Internet}

https://www.insee.fr/fr/statistiques/3526066?sommaire=3526086

https://www.insee.fr/fr/metadonnees/definition/c2139

https://www.education.gouv.fr/ site du Bulletin officiel du ministère de l'éducation nationale

LOI $\mathrm{n}^{\circ}$ 2018-771 du 5 septembre 2018 pour la liberté de choisir son avenir professionnel

NOR:

MTRX1808061L

ELI: https://www.legifrance.gouv.fr/eli/loi/2018/9/5/MTRX1808061L/jo/texte Alias: Titre ier: vers une nouvelle société de compétences. 